\title{
Rang de l'image du groupe des unités et polynômes lacunaires
}

\author{
par \\ Gabriele Ranieri (Caen et Pisa)
}

1. Introduction. Dans [Amo1] F. Amoroso a introduit la notion de corps proche d'un corps CM (en abrégé : PCM). Soit $K$ un corps de nombres et soit $\Gamma$ son groupe de $\mathbb{Q}$-automorphismes. Suivant [Amo1], nous dirons que $K$ est un corps $P C M$ s'il existe $\phi \in \mathbb{Z}[\Gamma]$ tel que

$$
\|\phi\|_{1} R_{\phi}<[K: \mathbb{Q}] \text {. }
$$

Ici $\|\phi\|_{1}$ est la taille de $\phi$ (i.e. si $\phi=\sum_{\sigma \in \Gamma} \phi_{\sigma} \sigma$ alors $\|\phi\|_{1}=\sum_{\sigma \in \Gamma}\left|\phi_{\sigma}\right|$ ), et $R_{\phi}=\operatorname{dim}\left(\mathcal{L}\left(K^{* \phi}\right) \otimes \mathbb{R}\right)$, où $\mathcal{L}$ est le plongement logarithmique et

$$
K^{* \phi}=\left\{\beta \in K^{*}: \exists \alpha \in K^{*}, \beta=\alpha^{\phi}\right\} .
$$

Remarquons que si $K$ est un corps $\mathrm{CM}$ et $j$ est la conjugaison complexe, alors $R_{1-j}\|1-j\|_{1}=0$, en particulier tout corps CM est PCM. D'autres exemples remarquables de corps PCM sont donnés par $K=\mathbb{Q}(\alpha)$, où $\alpha$ est un nombre de Salem (voir [Amo2]).

Le problème de caractériser les corps $\mathrm{PCM}$ se pose; nous donnons ici une réponse partielle, en montrant qu'une extension totalement réelle n'est jamais PCM (corollaire 1).

Soit maintenant $k$ un entier positif et soient $d_{1}, \ldots, d_{k} \in \mathbb{N}^{*}$. Dans le cas particulier des extensions abéliennes totalement réelles, le problème de déterminer les éventuels corps PCM se traduit, via la correspondance entre caractères et racines de l'unité (voir appendice), en l'existence de polynômes non nuls $P \in \mathbb{Z}\left[x_{1}, \ldots, x_{k}\right]$ de degrés partiels $\operatorname{deg}_{x_{i}}(P)<d_{i}$ pour tout $1 \leq$ $i \leq k$, tels que

$$
\|P\|_{1} R_{\mathbf{d}, P}<d_{1} \cdots d_{k} .
$$

Ici $\mathbf{d}=\left(d_{1}, \ldots, d_{k}\right),\|P\|_{1}$ est la somme des modules des coefficients de $P$ et

$$
R_{\mathbf{d}, P}=\left|\left\{\boldsymbol{\omega} \in \mu_{d_{1}} \times \cdots \times \mu_{d_{k}}: P(\boldsymbol{\omega}) \neq 0\right\}\right|
$$

(où $\mu_{d_{i}}$ est l'ensemble des racines $d_{i}$-ièmes de l'unité pour tout $1 \leq i \leq k$ ).

2000 Mathematics Subject Classification: 11C08, 11R04. 
F. Amoroso avait conjecturé que pour tout $P \neq 0$ dans $\mathbb{Z}\left[x_{1}, \ldots, x_{k}\right]$, on a

$$
\|P\|_{1} R_{\mathbf{d}, P} \geq d_{1} \cdots d_{k} .
$$

A. Schinzel a ensuite remarqué (communication orale) que même dans le cas très particulier

$$
P(x)=\frac{x^{n}-1}{\phi_{n}(x)} \in \mathbb{Z}[x]
$$

où $n$ est un entier positif et $\phi_{n}$ est le $n$-ième polynôme cyclotomique (donc, en suivant les notations précédentes, dans ce cas $k=1$ et $n=d_{1}$ ) cette conjecture n'était pas évidente.

Ici nous prouvons une version plus forte de la conjecture de F. Amoroso ; plus précisément (paragraphe 2), en utilisant des techniques élémentaires nous démontrons le

THÉORÈme 1. Soit $k$ un entier positif, soit $P \in \mathbb{C}\left[x_{1}, \ldots, x_{k}\right]$ un polynôme non nul tel que $\operatorname{deg}_{x_{i}}(P)<d_{i}$ pour tout $1 \leq i \leq k$ et soit $\Omega_{P}$ le nombre de coefficients $\neq 0$ de $P$. Alors

$$
\Omega_{P} \geq \frac{d_{1} \cdots d_{k}}{R_{\mathbf{d}, P}}
$$

Il est clair que la conjecture de F. Amoroso découle de (1). En effet, si $P \in \mathbb{Z}\left[x_{1}, \ldots, x_{k}\right]$ alors $\|P\|_{1} \geq \Omega_{P}$ car tout coefficient de $P$ non nul est de module $\geq 1$.

Remarquons que la minoration (1) est optimale. En effet, si $l, m$ sont entiers positifs tels que $l$ divise $m$ et si on pose

$$
G(x):=1+x^{l}+x^{2 l}+\cdots+x^{m-l}=\frac{x^{m}-1}{x^{l}-1},
$$

on a $\operatorname{deg}(G)<m, \Omega_{G}=m / l$ et $R_{m, G}=l$; donc

$$
\Omega_{G}=\frac{m}{R_{m, G}} \text {. }
$$

De même, si $v$ est un entier positif pair et si on pose

$$
H(x)=1-x^{v / 2},
$$

on a encore $\operatorname{deg}(H)<v, \Omega_{H}=2$ et $R_{v, H}=v / 2$; en particulier,

$$
\Omega_{H}=\frac{v}{R_{v, H}} .
$$

Dans le paragraphe 3 , nous généralisons la méthode du théorème 1 au cas de groupes non nécessairement commutatifs pour montrer

ThÉORÈme 2. Soit $G$ un groupe fini et soit $\phi=\sum_{\sigma \in G} \phi_{\sigma} \sigma$ un élément non nul de $\mathbb{C}[G]$; notons $\Omega_{\phi}$ le nombre des coefficients $\neq 0$ de $\phi$ et $\widetilde{R}_{\phi}$ le 
rang de l'application linéaire $T_{\phi}: \mathbb{C}[G] \rightarrow \mathbb{C}[G]$ qui envoie $\psi \in \mathbb{C}[G]$ sur $\psi \phi$. Alors

$$
\Omega_{\phi} \widetilde{R}_{\phi} \geq|G| .
$$

Le théorème 2 permet de répondre en toute généralité à la question de l'existence de corps PCM réels :

Corollaire 1. Aucun corps totalement réel n'est PCM.

Remerciements. Je tiens à remercier B. Anglès et B. Leclerc pour leur aide lors de la réalisation de cet article. Je tiens également à remercier P. Gillibert pour ses intéressantes remarques, et C. Pontreau qui a relu une version préliminaire de ce travail.

2. Preuve du théorème 1. Puisque $\operatorname{deg}_{x_{i}}(P)<d_{i}$ pour tout $1 \leq i \leq k$, nous avons

$$
P(\mathbf{x})=\sum_{h_{1}=0}^{d_{1}-1} \ldots \sum_{h_{k}=0}^{d_{k}-1} P_{\mathbf{h}} \mathbf{x}^{\mathbf{h}} \in \mathbb{C}\left[x_{1}, \ldots, x_{k}\right] .
$$

Montrons tout d'abord, pour tout $\mathbf{l} \in \mathbb{Z}^{k}$, l'égalité

$$
P_{\mathbf{l}}=\frac{1}{d} \sum_{\boldsymbol{\omega} \in \mu_{\mathbf{d}}} \overline{\boldsymbol{\omega}^{\mathbf{1}}} P(\boldsymbol{\omega}),
$$

où on a posé $d:=d_{1} \cdots d_{k}$ et $\mu_{\mathbf{d}}=\mu_{d_{1}} \times \cdots \times \mu_{d_{k}}$. D'après (2), pour tout $\boldsymbol{\omega} \in \mu_{\mathbf{d}}$, on a

$$
P(\boldsymbol{\omega})=\sum_{h_{1}=0}^{d_{1}-1} \cdots \sum_{h_{k}=0}^{d_{k}-1} P_{\mathbf{h}} \boldsymbol{\omega}^{\mathbf{h}} .
$$

Il vient

$$
\begin{aligned}
\frac{1}{d} \sum_{\boldsymbol{\omega} \in \mu_{\mathbf{d}}} \overline{\boldsymbol{\omega}^{\mathbf{l}}} P(\boldsymbol{\omega}) & =\frac{1}{d} \sum_{\boldsymbol{\omega} \in \mu_{\mathbf{d}}} \overline{\boldsymbol{\omega}^{\mathbf{l}}} \sum_{h_{1}=0}^{d_{1}-1} \cdots \sum_{h_{k}=0}^{d_{k}-1} P_{\mathbf{h}} \boldsymbol{\omega}^{\mathbf{h}} \\
& =\frac{1}{d} \sum_{\boldsymbol{\omega} \in \mu_{\mathbf{d}}} \sum_{h_{1}=0}^{d_{1}-1} \cdots \sum_{h_{k}=0}^{d_{k}-1} P_{\mathbf{h}} \boldsymbol{\omega}^{\mathbf{h}-\mathbf{l}} \\
& =\frac{1}{d} \sum_{h_{1}=0}^{d_{1}-1} \cdots \sum_{h_{k}=0}^{d_{k}-1} \sum_{\boldsymbol{\omega} \in \mu_{\mathbf{d}}} P_{\mathbf{h}} \boldsymbol{\omega}^{\mathbf{h}-\mathbf{l}} .
\end{aligned}
$$

Soit maintenant $\mathbf{h} \in \mathbb{Z}^{k}$. Si $\mathbf{h}=\mathbf{l}$ on a $\boldsymbol{\omega}^{\mathbf{h}-\mathbf{l}}=1$ et

$$
\frac{1}{d} \sum_{\omega \in \mu_{\mathrm{d}}} P_{1} \omega^{1-1}=P_{1}
$$


Par ailleurs si $\mathbf{h}-\mathbf{l}=\mathbf{m} \neq \mathbf{0}$ on a

$$
\frac{1}{d} \sum_{\boldsymbol{\omega} \in \mu_{\mathbf{d}}} P_{\mathbf{h}} \boldsymbol{\omega}^{\mathbf{m}}=0
$$

Des relations (5) et (6) il vient que

$$
\frac{1}{d} \sum_{h_{1}=0}^{d_{1}-1} \cdots \sum_{h_{k}=0}^{d_{k}-1} \sum_{\boldsymbol{\omega} \in \mu_{\mathbf{d}}} P_{\mathbf{h}} \boldsymbol{\omega}^{\mathbf{h}-\mathbf{1}}=P_{\mathbf{l}}
$$

d'où l'égalité (3).

Choisissons maintenant $\mathbf{M} \in \mathbb{Z}^{k}$ tel que pour tout vecteur $\mathbf{h}$ on ait $\left|P_{\mathbf{M}}\right| \geq\left|P_{\mathbf{h}}\right|$. Par la relation (3) on a

$$
P_{\mathbf{M}}=\frac{1}{d} \sum_{\boldsymbol{\omega} \in \mu_{\mathbf{d}}} \overline{\boldsymbol{\omega}^{\mathbf{M}}} P(\boldsymbol{\omega})
$$

Donc

$$
\left|P_{\mathbf{M}}\right| \leq \frac{1}{d} \sum_{\boldsymbol{\omega} \in \mu_{\mathbf{d}}}\left|\overline{\boldsymbol{\omega}^{\mathbf{M}}} P(\boldsymbol{\omega})\right|=\frac{1}{d} \sum_{\boldsymbol{\omega} \in \mu_{\mathbf{d}}}|P(\boldsymbol{\omega})| .
$$

En rappelant que, par définition, $R_{\mathbf{d}, P}$ est le nombre de $\boldsymbol{\omega} \in \mu_{\mathbf{d}}$ tels que $P(\boldsymbol{\omega})$ soit non nul et en observant que

$$
|P(\boldsymbol{\omega})| \leq \Omega_{P}\left|P_{\mathbf{M}}\right|
$$

(car $\left|\boldsymbol{\omega}^{\mathbf{h}}\right|=1$ pour tous $\boldsymbol{\omega}$ et $\mathbf{h}$ ), par la relation (7) on obtient

$$
\left|P_{\mathbf{M}}\right| \leq \frac{1}{d} \Omega_{P}\left|P_{\mathbf{M}}\right| R_{\mathbf{d}, P}
$$

Puisque par hypothèse $P$ est non nul, $P_{\mathbf{M}} \neq 0$ et donc on peut diviser les membres de la relation (8) par $\left|P_{\mathbf{M}}\right|$. On obtient alors le résultat souhaité.

3. Corps totalement réels et algèbres sur groupes finis. Comme déjà annoncé, dans ce paragraphe on montre que tout corps totalement réel n'est pas PCM.

Lemme 1. Soit $K$ un corps de nombres et soit $\Gamma$ son groupe de $\mathbb{Q}$ automorphismes; notons $L$ la clôture galoisienne de $K$ et posons $G:=$ $\operatorname{Gal}(L / \mathbb{Q})$. Alors, en utilisant les notations du paragraphe 1 , pour tout $\phi \in$ $\mathbb{Z}[\Gamma]$ il existe $\psi \in \mathbb{Z}[G]$ tel que

$$
\frac{\|\psi\|_{1} R_{\psi}}{[L: \mathbb{Q}]} \leq \frac{\|\phi\|_{1} R_{\phi}}{[K: \mathbb{Q}]}
$$

Preuve. Soit $\phi=\sum_{\sigma \in \Gamma} \phi_{\sigma} \sigma \in \mathbb{Z}[\Gamma]$. Pour tout $\sigma \in \Gamma$ choisissons un élément $\widetilde{\sigma} \in G$ tel que $\widetilde{\sigma}$ coïncide avec $\sigma$ sur $K$. Notons

$$
\widetilde{\phi}=\sum_{\sigma \in \Gamma} \phi_{\sigma} \widetilde{\sigma} \in \mathbb{Z}[G]
$$


Soit maintenant $H_{K}=\operatorname{Gal}(L / K)$ et posons $N=\sum_{\sigma \in H_{K}} \sigma$ et $\psi=\widetilde{\phi} N$. Il est alors évident, par construction, que $R_{\psi}=R_{\phi}$. De plus, puisque $\|\phi\|_{1}=$ $\|\widetilde{\phi}\|_{1}$ et $\|N\|_{1}=\left|H_{K}\right|=[L: K]$, nous obtenons

$$
\|\psi\|_{1} \leq \sum_{\sigma \in H_{K}}\|\widetilde{\phi} \sigma\|_{1}=\|\phi\|_{1}[L: K] .
$$

Donc, en observant que $[L: \mathbb{Q}]=[K: \mathbb{Q}][L: K]$ et que $R_{\psi}=R_{\phi}$, on en déduit

$$
\frac{\|\psi\|_{1} R_{\psi}}{[L: \mathbb{Q}]} \leq \frac{\|\phi\|_{1} R_{\phi}[L: K]}{[L: K][K: \mathbb{Q}]}=\frac{\|\phi\|_{1} R_{\phi}}{[K: \mathbb{Q}]}
$$

LEMme 2. Soit $K / \mathbb{Q}$ une extension galoisienne finie de $\mathbb{Q}$ totalement réelle de groupe de Galois $G$. Alors, en utilisant les notations du premier paragraphe, pour tout $\phi=\sum_{\sigma \in G} \phi_{\sigma} \sigma \in \mathbb{Z}[G]$ on $a: R_{\phi}=\widetilde{R}_{\phi}$.

Preuve. Puisque $K$ est, par hypothèse, un corps totalement réel, les places infinies de $K$ sont exactement les éléments de $G$. Donc, pour tout $\alpha \in K^{*}$,

$$
\mathcal{L}(\alpha)=\left(\log |\alpha|_{v}\right)_{v \mid \infty}=\left(\log \left|\alpha^{\sigma}\right|\right)_{\sigma \in G}
$$

Soit $L_{\phi}$ l'endomorphisme linéaire de $\mathcal{L}\left(K^{*}\right) \otimes \mathbb{R}$ défini par

$$
L_{\phi}\left(\left(\log \left|\alpha^{\sigma}\right|\right)_{\sigma} \otimes c\right)=\left(\log \left|\alpha^{\sigma \phi}\right|\right)_{\sigma} \otimes c
$$

pour tout $\alpha \in K^{*}$ et $c \in \mathbb{R}$. Puisque l'image de $L_{\phi}$ coïncide avec l'espace $\mathcal{L}\left(K^{* \phi}\right) \otimes \mathbb{R}$, le rang de $L_{\phi}$ est égal à $R_{\phi}$.

Rappelons maintenant que $\phi$ est un élément de $\mathbb{Z}[G]$. La multiplication à droite par $\phi$ est donc un endomorphisme linéaire de $\mathbb{Q}[G]$ et, par simples arguments d'algèbre linéaire, on obtient que la dimension du noyau d'un tel endomorphisme est égale à $\operatorname{dim}\left(\operatorname{ker}\left(T_{\phi}\right)\right.$ ) (on a utilisé la notation du théorème 2 où $T_{\phi}$ est défini comme l'endomorphisme linéaire de $\mathbb{C}[G]$ qui agit sur les éléments de $\mathbb{C}[G]$ en multipliant à droite par $\phi)$. Par ailleurs, une famille d'éléments de $\mathbb{Z}[G]$ êtant $\mathbb{Z}$-libre si et seulement si elle est $\mathbb{Q}$-libre, nous obtenons que $\operatorname{dim}\left(\operatorname{ker}\left(T_{\phi}\right)\right)$ est égal au rang du sous-module de $\mathbb{Z}[G]$ des éléments $\lambda$ tels que $\lambda \phi=0$. Notons maintenant $n=\operatorname{dim}\left(\operatorname{ker}\left(T_{\phi}\right)\right)$ et soit $C$ un ensemble de cardinalité maximale d'éléments $\lambda_{i}=\sum_{\sigma \in G} \lambda_{i, \sigma} \sigma \in \mathbb{Z}[G]$ indépendants sur $\mathbb{Z}$ tels que $\lambda_{i} \phi=0$ pour tout $1 \leq i \leq n$. Remarquons alors que $\mathcal{L}\left(K^{* \phi}\right) \otimes \mathbb{R}$ et

$$
V:=\left\{\mathbf{x} \in \mathcal{L}\left(K^{*}\right) \otimes \mathbb{R}: \sum_{\sigma \in G} \lambda_{i, \sigma} x_{\sigma}=0 \forall i\right\}
$$

coïncident. En effet, pour tout $\alpha \in K^{*}$ nous avons $\log \left|\alpha^{\lambda_{i} \phi}\right|=0$ pour tout $1 \leq i \leq n$. Donc $V$ contient $\mathcal{L}\left(K^{* \phi}\right) \otimes \mathbb{R}$. L'autre inclusion découle de la maximalité du cardinal de $C$. Par ailleurs, puisque les éléments $\lambda_{i}$ sont 
indépendants pour tout $1 \leq i \leq n$ et $n=\operatorname{dim}\left(\operatorname{ker}\left(T_{\phi}\right)\right)$, on obtient

$$
R_{\phi}=\operatorname{dim}\left(\mathcal{L}\left(K^{* \phi}\right) \otimes \mathbb{R}\right)=\operatorname{dim}(V)=|G|-\operatorname{dim}\left(\operatorname{ker}\left(T_{\phi}\right)\right)=\widetilde{R}_{\phi} .
$$

Le lemme 2 nous dit que le rang $R_{\phi}$ de $\mathcal{L}\left(K^{\phi}\right) \otimes \mathbb{R}$ est égal au rang $\widetilde{R}_{\phi}$ de l'endomorphisme $T_{\phi}$ de $\mathbb{C}[G]$ qui envoie $\psi \in \mathbb{C}[G]$ sur $\psi \phi$. Dans la suite on notera donc également $R_{\phi}$ le rang de $T_{\phi}$.

Le lemme suivant est le dernier résultat nécessaire pour la preuve du théorème 2 .

Lemme 3. Soient $n, r$ entiers positifs et soient $U_{1}, \ldots, U_{n}$ matrices unitaires d'ordre $r \times r$ à coefficients dans $\mathbb{C}$. De plus, soient $\lambda_{1}, \ldots, \lambda_{n} \in \mathbb{C}$ et posons $M=\sum_{i=1}^{n} \lambda_{i} U_{i}$. Alors

$$
|\operatorname{Tr}(M)| \leq\left(\sum_{i=1}^{n}\left|\lambda_{i}\right|\right) R(M)
$$

où $\operatorname{Tr}(M)$ est la trace de $M$ et $R(M)$ son rang.

Preuve. Puisque la trace de $M$ est égale à la somme de ses valeurs propres et le rang de $M$ est égal au nombre de ses valeurs propres non nuls (toute valeur propre est comptée avec sa multiplicité algébrique), si $\mu$ est une valeur propre de module maximum on a

$$
|\operatorname{Tr}(M)| \leq|\mu| R(M) .
$$

Il suffit donc de montrer l'inégalité

$$
|\mu| \leq \sum_{i=1}^{n}\left|\lambda_{i}\right|
$$

Par hypothèse pour tout $1 \leq i \leq n$ les matrices $U_{i}$ sont unitaires. Donc, en considérant la norme $\|\cdot\|$ induite par le produit scalaire standard de $\mathbb{C}^{r}$, on a, pour tout $v \in \mathbb{C}^{r}$,

$$
\left\|U_{i}(v)\right\|=\|v\| .
$$

Soit maintenant $w$ un vecteur propre de $M$ de norme 1 associé à la valeur propre $\mu$. On obtient

$$
|\mu|=\|M(w)\|=\left\|\sum_{i=1}^{n} \lambda_{i} U_{i}(w)\right\| \leq \sum_{i=1}^{n}\left\|\lambda_{i} U_{i}(w)\right\|=\sum_{i=1}^{n}\left|\lambda_{i}\right| .
$$

Avant de terminer ce paragraphe avec la preuve du théorème 2 et du corollaire 1 , rappelons quelques propriétés des $\mathbb{C}$-algèbres sur groupes finis.

Soit $G$ un groupe fini, soit $\operatorname{Irr}(G)$ l'ensemble des caractères irréductibles de $G$ et considérons la $\mathbb{C}$-algèbre $\mathbb{C}[G]$. La théorie des représentations (voir [Isa, Chapter 1]) nous dit que

$$
\mathbb{C}[G]=\bigoplus_{\chi \in \operatorname{Irr}(G)} I_{\chi}^{\chi(1)}
$$


où $I_{\chi}$ est un espace vectoriel sur $\mathbb{C}$ de dimension $\chi(1)$ et un idéal à droite de $\mathbb{C}[G]$.

Soit maintenant $\phi \in \mathbb{C}[G]$. Considérons l'endomorphisme $T_{\phi}$ précédemment défini (par exemple voir l'énoncé du théorème 2 dans le premier paragraphe). Puisque pour tout caractère $\chi$ l'ensemble $I_{\chi}$ est un idéal à droite, la restriction $T_{\chi, \phi}$ de $T_{\phi}$ à $I_{\chi}$ est une application de $I_{\chi}$ dans $I_{\chi}$. De plus, si $R_{\phi}$ désigne le rang de $T_{\phi}$ et $R_{\chi, \phi}$ celui de $T_{\chi, \phi}$, par (9) on a

$$
R_{\phi}=\sum_{\chi \in \operatorname{Irr}(G)} \chi(1) R_{\chi, \phi}
$$

Preuve du théorème 2. Soit $\phi=\sum_{\sigma \in G} \phi_{\sigma} \sigma$ un élément non nul de $\mathbb{C}[G]$. Posons

$$
\|\phi\|_{1}=\sum_{\sigma \in G}\left|\phi_{\sigma}\right| .
$$

Pour tout $\sigma \in G$ on a $\|\phi\|_{1}=\|\phi \sigma\|_{1}, R_{\phi}=R_{\phi \sigma}$ et $\Omega_{\phi}=\Omega_{\phi \sigma}$ (rappelons que nous avons changé la notation après le lemme 2 en posant $\widetilde{R}_{\phi}=R_{\phi}$ ). Nous pouvons donc supposer $\left|\phi_{1}\right| \geq\left|\phi_{\sigma}\right|$ pour tout $\sigma \in G$. Par conséquent,

$$
\|\phi\|_{1} \leq \Omega_{\phi}\left|\phi_{1}\right| \text {. }
$$

Définissons maintenant le nombre complexe

$$
\beta_{\phi}=\sum_{\chi \in \operatorname{Irr}(G)} \chi(1) \sum_{\sigma \in G} \phi_{\sigma} \chi(\sigma) .
$$

Démontrons tout d'abord que

$$
\left|\beta_{\phi}\right| \leq\|\phi\|_{1} R_{\phi}
$$

Soit $\chi \in \operatorname{Irr}(G)$ et fixons une base $B_{\chi}$ de $I_{\chi}$. Pour tout $\psi \in \mathbb{C}[G]$ nous pouvons associer à l'application linéaire $T_{\chi, \psi} \in \operatorname{End}\left(I_{\chi}\right)$ qui envoie $\alpha \in I_{\chi}$ sur $\alpha \psi$, la matrice $M_{\chi, \psi}$ de $T_{\chi, \psi}$ dans la base $B_{\chi}$. En particulier, pour tout $\sigma \in G$ les matrices $M_{\chi, \sigma}$ sont bien définies. De plus

$$
M_{\chi, \phi}=\sum_{\sigma \in G} \phi_{\sigma} M_{\chi, \sigma}
$$

Par définition de caractère, $\chi(\sigma)$ est la trace de la matrice $M_{\chi, \sigma}$; en outre, les matrices $M_{\chi, \sigma}$ sont unitaires car $T_{\chi, \sigma}$ est d'ordre fini. On peut donc appliquer le lemme 3 à la matrice $M_{\chi, \phi}$, ce qui donne

$$
\left|\sum_{\sigma \in G} \phi_{\sigma} \chi(\sigma)\right|=\left|\operatorname{Tr}\left(M_{\chi, \phi}\right)\right| \leq\|\phi\|_{1} R_{\chi, \phi} .
$$

Par cette relation et par (10) on a

$$
\left|\beta_{\phi}\right| \leq \sum_{\chi \in \operatorname{Irr}(G)} \chi(1)\left|\sum_{\sigma \in G} \phi_{\sigma} \chi(\sigma)\right| \leq\|\phi\|_{1} R_{\phi} .
$$


Calculons maintenant la valeur de $\beta_{\phi}$ à l'aide des lois d'orthogonalité entre les colonnes des tables des caractères (voir [Isa, (2.13) Theorem et (2.14) Corollary])

$$
\beta_{\phi}=\sum_{\chi \in \operatorname{Irr}(G)} \chi(1) \sum_{\sigma \in G} \phi_{\sigma} \chi(\sigma)=\sum_{\sigma \in G} \phi_{\sigma} \sum_{\chi \in \operatorname{Irr}(G)} \chi(\sigma) \chi(1)=\phi_{1}|G| .
$$

On en déduit que

$$
\|\phi\|_{1} R_{\phi} \geq\left|\phi_{1}\right||G|
$$

et, puisque $\Omega_{\phi}\left|\phi_{1}\right| \geq\|\phi\|_{1}$ et $\phi_{1} \neq 0$ car $\phi$ est non nul, on obtient le théorème.

Preuve du corollaire 1. Soit $K$ un corps totalement réel. Puisque, par le lemme 1, si un corps est PCM alors sa clôture galoisienne est PCM, on peut supposer l'extension $K / \mathbb{Q}$ galoisienne. Notons alors $G$ le groupe de Galois de $K / \mathbb{Q}$ et soit $\phi$ un élément non nul de $\mathbb{Z}[G]$. Par le lemme 2 la dimension $R_{\phi}$ de $\mathcal{L}\left(K^{\phi}\right) \otimes \mathbb{R}$ est égale au rang de l'endomorphisme de $\mathbb{C}[G]$ qui envoie $\psi \in \mathbb{C}[G]$ sur $\psi \phi$. Par ailleurs, par la remarque précédente et le théorème 2 on a

$$
\Omega_{\phi} R_{\phi} \geq|G| .
$$

Puisque les coefficients de $\phi$ sont entiers, nous avons $\Omega_{\phi} \leq\|\phi\|_{1}$. De plus, $|G|=[K: \mathbb{Q}]$ car $K / \mathbb{Q}$ est une extension de galoisienne. Donc

$$
\|\phi\|_{1} R_{\phi} \geq[K: \mathbb{Q}]
$$

et $K$ n'est pas PCM.

4. Appendice. Soit $G$ un groupe abélien fini et soient $d_{1}, \ldots, d_{k}$ entiers positifs tels que $G$ soit isomorphe à $\mathbb{Z} / d_{1} \mathbb{Z} \times \cdots \times \mathbb{Z} / d_{k} \mathbb{Z}$ (après on identifiera $G$ avec le groupe $\mathbb{Z} / d_{1} \mathbb{Z} \times \cdots \times \mathbb{Z} / d_{k} \mathbb{Z}$ et $\left\{0,1, \ldots, d_{i}-1\right\}$ avec $\mathbb{Z} / d_{i} \mathbb{Z}$ pour $1 \leq i \leq k)$. Soit $F: \mathbb{C}[G] \rightarrow \mathbb{C}\left[x_{1}, \ldots, x_{k}\right]$ l'application qui envoie

$$
\phi=\sum_{\mathbf{h} \in G} \phi_{\mathbf{h}} \mathbf{h} \in \mathbb{C}[G]
$$

sur

$$
F(\phi)=\sum_{h_{1}=0}^{d_{1}-1} \cdots \sum_{h_{k}=0}^{d_{k}-1} \phi_{\mathbf{h}} \mathbf{x}^{\mathbf{h}} .
$$

Il est bien évident que $F$ définit une correspondance biunivoque entre les éléments de $\mathbb{C}[G]$ et les polynômes $Q \in \mathbb{C}\left[x_{1}, \ldots, x_{k}\right]$ tels que $\operatorname{deg}_{x_{i}}(Q)<d_{i}$ pour tout $1 \leq i \leq k$.

Fixons maintenant $\phi \in \mathbb{C}[G]$ et notons $P\left(x_{1}, \ldots, x_{k}\right):=F(\phi)$. Par définition de $F$ il suit immédiatement que $\Omega_{\phi}=\Omega_{P}$. Nous voulons montrer que même $R_{\phi}$ est égal à $R_{\mathbf{d}, P}$. 
Soit $\chi \in \operatorname{Irr}(G)$. Puisque $G$ est abélien, $\operatorname{Irr}(G)$ est un groupe isomorphe à $G$ et $\chi(1)=1$. De plus, $R_{\chi, \phi}=0$ si et seulement si

$$
\sum_{\mathbf{h} \in G} P_{\mathbf{h}} \chi(\mathbf{h})=0
$$

(voir [Was, p. 100]). On a donc

$$
R_{\phi}=\left|\left\{\chi \in \operatorname{Irr}(G): \sum_{\mathbf{h} \in G} P_{\mathbf{h}} \chi(\mathbf{h}) \neq 0\right\}\right| .
$$

Soit maintenant $\boldsymbol{\omega}=\left(\omega_{1}, \ldots, \omega_{k}\right) \in \mu_{d_{1}} \times \cdots \times \mu_{d_{k}}$. Il est bien évident que la fonction $\chi_{\boldsymbol{\omega}}: G \rightarrow \mathbb{C}^{*}$ qui envoie $\mathbf{h}=\left(h_{1}, \ldots, h_{k}\right)$ sur $\prod_{i=1}^{k} \omega_{i}^{h_{i}}$ est un caractère irréductible de $G$. De plus, on obtient

$$
\sum_{\mathbf{h} \in G} P_{\mathbf{h}} \chi_{\boldsymbol{\omega}}(\mathbf{h})=\sum_{\mathbf{h} \in G} P_{\mathbf{h}} \omega^{\mathbf{h}}
$$

et donc $R_{\chi_{\boldsymbol{\omega}}, \phi}=0$ si et seulement si $P(\boldsymbol{\omega})=0$. D'ailleurs, si $\chi \in \operatorname{Irr}(G)$ alors

$$
\boldsymbol{\omega}_{\chi}:=(\chi(1,0, \ldots, 0), \ldots, \chi(0,0, \ldots, 1))
$$

appartient à $\mu_{d_{1}} \times \cdots \times \mu_{d_{k}}$. De plus, par construction,

$$
\sum_{\mathbf{h} \in G} P_{\mathbf{h}} \omega_{\chi}^{\mathbf{h}}=\sum_{\mathbf{h} \in G} P_{\mathbf{h}} \chi(\mathbf{h}) .
$$

Puisque $\operatorname{Irr}(G)$ est isomorphe à $G$, qui à son tour est isomorphe à $\mu_{d_{1}} \times$ $\cdots \times \mu_{d_{k}}$, la correspondance que nous venons de définir entre les caractères irréductibles de $G$ et les éléments de $\mu_{d_{1}} \times \cdots \times \mu_{d_{k}}$ est biunivoque. On a alors

$$
\left|\left\{\chi \in \operatorname{Irr}(G): \sum_{\mathbf{h} \in G} P_{\mathbf{h}} \chi(\mathbf{h}) \neq 0\right\}\right|=\left|\left\{\boldsymbol{\omega} \in \mu_{d_{1}} \times \cdots \times \mu_{d_{k}}: P(\boldsymbol{\omega}) \neq 0\right\}\right|
$$

et, par (12) et par définition de $R_{\mathbf{d}, P}$, on obtient

$$
R_{\phi}=R_{\mathbf{d}, P} .
$$

En conclusion, on a donc montré qu'il est possible de définir une correspondance biunivoque entre les éléments $\phi$ de $\mathbb{C}[G]$ (où $G$ est isomorphe au groupe $\left.\mathbb{Z} / d_{1} \mathbb{Z} \times \cdots \times \mathbb{Z} / d_{k} \mathbb{Z}\right)$ et les polynômes $P$ de $\mathbb{C}\left[x_{1}, \ldots, x_{k}\right]$ tels que $\operatorname{deg}_{x_{i}}(P)<d_{i}$ pour tout $1 \leq i \leq k$. De plus, si $P$ correspond à $\phi$ nous avons que $\Omega_{\phi}=\Omega_{P}$ et $R_{\phi}=R_{\mathbf{d}, P}$.

\section{Références}

[Amo1] F. Amoroso, Groupes des classes de corps « proches » d'un corps CM, preprint, 2005.

[Amo2] - Une minoration pour l'exposant du groupe des classes d'un corps engendré par un nombre de Salem, J. Number Theory, à paraître. 
[Isa] I. M. Isaacs, Character Theory of Finite Groups, 1st ed., Pure Appl. Math. 69, Academic Press, New York, 1976.

[Was] L. C. Washinghton, Introduction to Cyclotomic Fields, 2nd ed., Grad. Texts in Math. 83, Springer, New York, 1997.

Laboratoire de mathématiques

Nicolas Oresme, CNRS UMR 6139

Université de Caen, BP 5186

14032 Caen Cedex, France

E-mail: ranieri@math.unicaen.fr
Dipartimento di Matematica Leonida Tonelli Largo Bruno Pontecorvo, 5 56127 Pisa, Italy E-mail: ranieri@mail.dm.unipi.it

Reçu le 17.6.2006

et révisé le 7.10.2006 